NSA-95-1

\title{
Calculation of Beam Neutralization in the IPNS-Upgrade RCS
}

\author{
Yong-Chul Chae \\ Accelerator Systems Division \\ Argonne National Laboratory \\ 9700 S. Cass Ave., Argonne, IL 60439 \\ January 26,1995
}

\begin{abstract}
We calculated the neutralization of circulating beam in this report. In the calculation we assumed that all electrons liberated from the background molecules due to the collisional processes are trapped in the potential well of the proton beam. Including the dependence of ionization cross sections on the kinetic energy of the incident particle, we derived the empirical formula for beam neutralization as a function of time and baseline vacuum pressure, which is applicable to the one acceleration cycle of the IPNS-Upgrade RCS.
\end{abstract}




\section{DISCLAIMER}

This report was prepared as an account of work sponsored by an agency of the United States Government. Neither the United States Government nor any agency thereof, nor any of their employees, make any warranty, express or implied, or assumes any legal liability or responsibility for the accuracy, completeness, or usefulness of any information, apparatus, product, or process disclosed, or represents that its use would not infringe privately owned rights. Reference herein to any specific commercial product, process, or service by trade name, trademark, manufacturer, or otherwise does not necessarily constitute or imply its endorsement, recommendation, or favoring by the United States Government or any agency thereof. The views and opinions of authors expressed herein do not necessarily state or reflect those of the United States Government or any agency thereof. 


\section{DISCLAIMER}

Portions of this document may be illegible in electronic image products. Images are produced from the best available original document. 


\section{Introduction}

When the proton beam is circulating in the ring, the residual gas in the vacuum chamber is ionized due to the collisional process. The electrons liberated from the molecules then can be trapped in the potential well provided by the proton beam. An effect of the neutralization [1] by the trapped electrons is to reduce the space-charge tune shift, which is often desirable in operating the synchrotron. The other effects will include the coupled oscillation of electrons and protons through the potential well. In the analysis of these effects, the neutralization of the beam needs to be determined, which is the subject of this report.

\section{Ionization}

The collisions between charged particles and atoms result partly in excitation, partly in ionization of the atoms. If the impact parameter, the distance of closest approach, is large compared with the dimension of the atom, the atom reacts as a whole mainly resulting in the excitation of the atom. If the momentum transfer to the atomic electron by the passing particle is larger than the ionization of energy of atom, such an electron will be ejected from the atom leaving the atom ionized. This collisional process is often called distant collision. In general, the kinetic energy of a freed electron is small compared with the incident particle.

If the distance of closest approach is of the order of the atomic dimension, the interaction is mainly between the incident particle and one of the atomic electrons. As long as the interaction time is short compared with any atomic time associated with orbital motion of an atomic electron, the atomic electron can be treated as a free electron. As a consequence of the interaction, the electron is ejected from the atom with considerable energy. We may call this process close collision.

Both of the processes described above can result in the ionization of an atom. The theory of primary ionization, which is defined as the average number of collisions per $\mathrm{cm}$ of path that result in the ejection of an electron from an atom, was developed by Bethe. The result in terms of cross section is expressed by the equation [2]:

$$
\sigma=2 \pi r_{e}^{2} m_{e} c^{2} z^{2} \frac{Z a}{I_{0}} \frac{1}{\beta^{2}}\left[\ln \frac{2 m_{e} c^{2} \beta^{2}}{\left(1-\beta^{2}\right) I_{0}}+s-\beta^{2}\right],
$$

where $\sigma=$ cross section for the primary ionization $\left(\mathrm{cm}^{2}\right), r_{e}=e^{2} / m c^{2}$ (classical electron radius), $m_{e}=$ electron rest mass, $c=$ velocity of light, $\beta=$ ratio of incident particle velocity to the velocity of light, $z=$ incident particle charge, $Z=$ atomic number of the gas, $I_{0}=$ ionization potential of the gas, and $a, b=$ dimensionless constants dependent upon the electronic structure of the atom. For the particular 
case of atomic hydrogen $\left(I_{0}=13.5 \mathrm{eV}, Z=1\right)$, Bethe has calculated the values of the constants in this equation to be: $a=0.285, b=3.04$.

The ionization cross sections of gaseous molecules were measured. In the experimental study [3], the following formula is used

$$
\sigma=4 \pi\left(\hbar / m_{e} c\right)^{2}\left(M^{2} x_{1}+C x_{2}\right)
$$

where

$$
\begin{aligned}
4 \pi\left(\frac{\hbar}{m_{e} c}\right)^{2} & =1.87410^{-24-} m^{2} \\
x_{1} & =\frac{1}{\beta^{2}} \ln \left(\frac{\beta^{2}}{1-\beta^{2}}\right)-1 \\
x_{2} & =\frac{1}{\beta^{2}}
\end{aligned}
$$

$M^{2}$ and $C$ are characteristics of the gaseous molecules. The quantity $M^{2}$ is measured in units of $a_{0}^{2}=\left(\hbar^{2} / m_{e} e^{2}\right)^{2}$, the Bohr radius squared. These two constants are listed in Table 1 together with the atomic number of the gas, $Z$.

\begin{tabular}{|c|c|c|c|}
\hline Name of Molecule & $M^{2}$ & $C$ & $Z$ \\
\hline $\mathrm{C}_{6} \mathrm{H}_{4}$ & 17.5 & 162.4 & 46 \\
$\mathrm{CO}_{2}$ & 5.75 & 55.9 & 22 \\
$\mathrm{O}_{2}$ & 4.2 & 38.8 & 16 \\
$\mathrm{CO}$ & 3.7 & 35.1 & 14 \\
$\mathrm{~N}_{2}$ & 3.7 & 34.8 & 14 \\
$\mathrm{H}_{2} \mathrm{O}$ & 3.2 & 32.3 & 10 \\
$\mathrm{H}_{2}$ & 0.5 & 8.1 & 2 \\
\hline
\end{tabular}

Table 1: Measured values of $M^{2}$ and $C$.

Since ionization cross section is a function of incident particle's velocity, we investigate the variation of cross section against injection energy of beams. The result is shown in Fig. 1 and Fig. 2 with the energy range appropriate for the IPNS-Upgrade RCS acceleration cycle. The cross section at the ejection energy $(\mathrm{T}=2.2 \mathrm{GeV})$ is smaller by $25 \%$ than the one at the injection energy $(\mathrm{T}=400 \mathrm{MeV})$.

The ionization rate is defined as

$$
\tau_{m}^{-1}=d_{m} \sigma_{m} \beta c
$$


where $d_{m}$ is the molecular density, which in turn is related to the partial pressure by

$$
d_{m}\left(m^{-3}\right)=9.65 \times 10^{24} \frac{P_{m}(\text { Torr })}{T\left({ }^{\circ} K\right)} .
$$

Assuming the vacuum chamber is at the room temperature, and using $N_{2}$ pressure as the base pressure, the neutralization of beam during the acceleration cycle is shown in Fig. 3. From which we derive an empirical formula:

$$
\eta=0.842 P(n \text { Torr }) t(s e c) .
$$

In deriving the above formula, we assumed that the ionization rate is equal to the neutralization, which is a good approximation if the circulating beam is unbunched (coasting beam). If the beam is bunched, the local electrons experience the periodic forces in time as the proton bunches pass by. The proton bunches act as an alternating gradient (AG) focusing force on the electrons. Thus the effective neutralization rate. is in general significantly less than the ionization rate, or even zero. The estimate of the effective neutralization is the subject of the next report.

\section{Conclusion}

In this report, we estimated the beam neutralization in the IPNS-Upgrade RCS. The final result was expressed as functions of the vacuum pressure and time, which is valid for the envisioned RCS operational cycle. However, AG focusing effects of the circulating bunch are not included in the estimation. The results including such effects will be reported separately.

\section{References}

[1] Y. Baconnier, "Neutralization of Accelerator Beams by Ionization of the Residual Gas," Proc. CERN Accelerator School CERN 85-19, Vol. 1 (1985), 267-300.

[2] B. Rossi, High-Energy Particles. Prentice-Hall Inc. (1952).

[3] F. F. Rieke and W. Prepejchal, "Ionization Cross Section of Gaseous Atoms and Molecules for High-Energy Electrons and Positrons," Phys. Rev., A 6, No. 4 (1972), 1507-1519. 

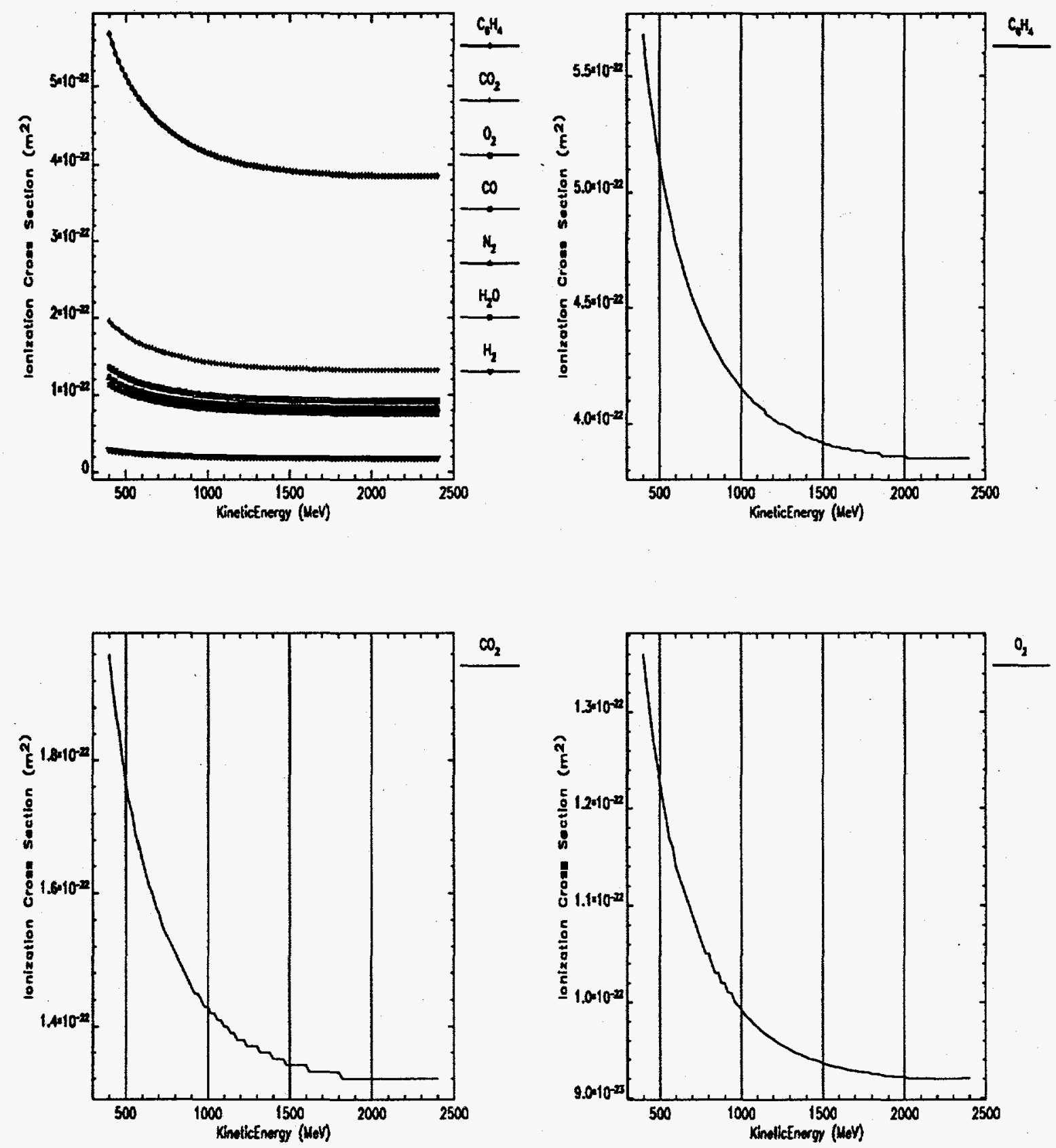

Figure 1: Ionization cross section for the gaseous molecules. 

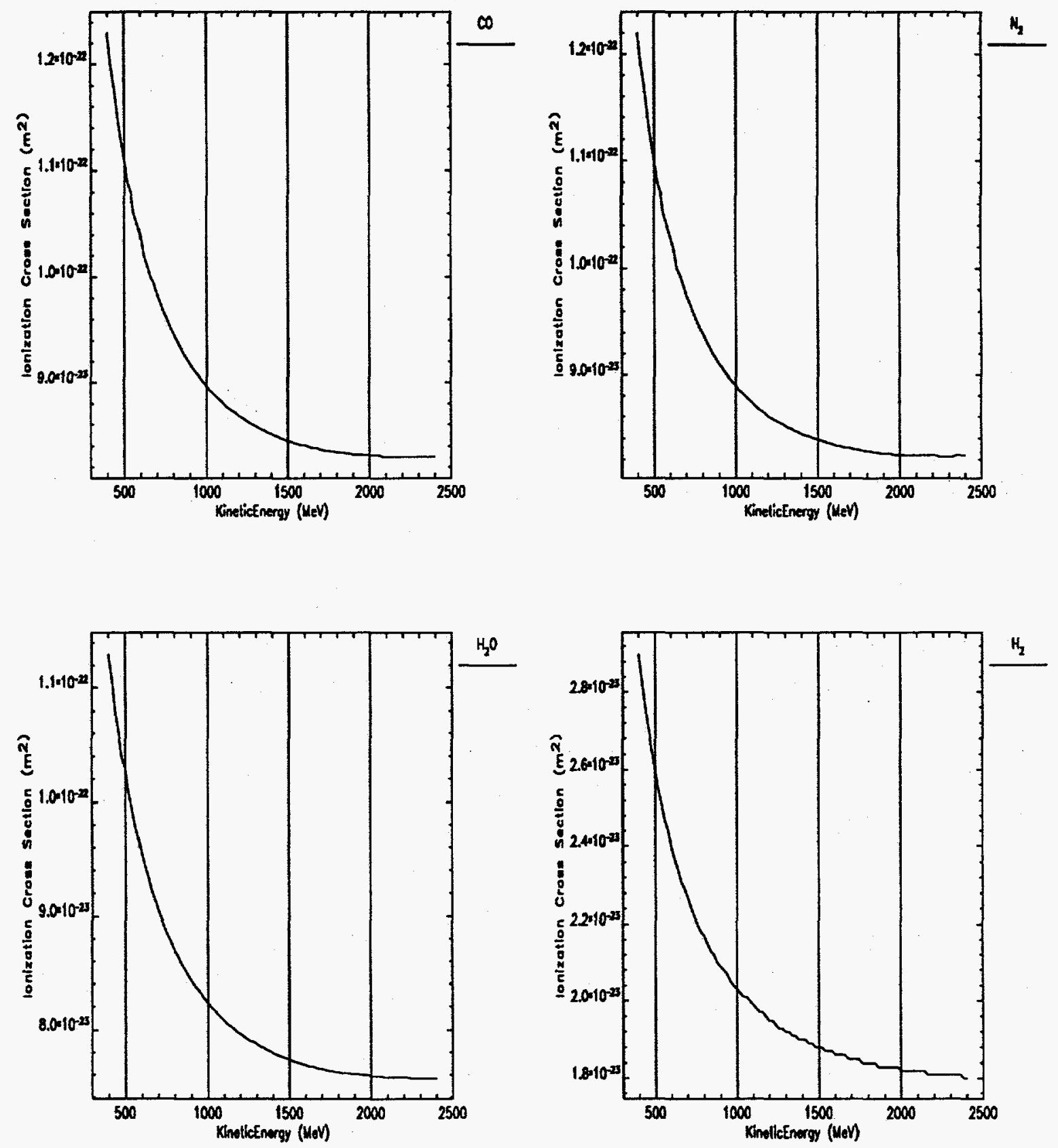

Figure 2:- Ionization cross section for the gaseous molecules. 
(0)

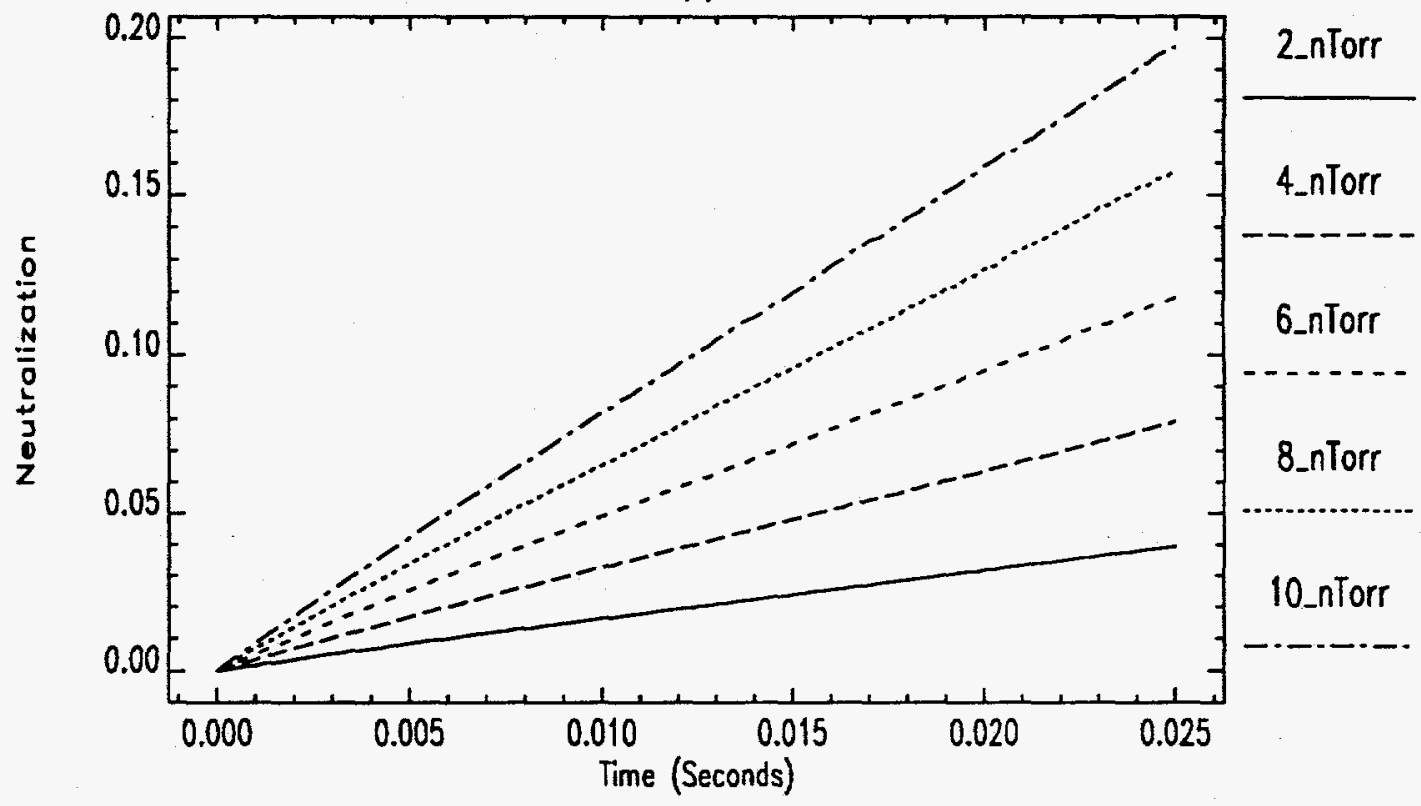

(b)

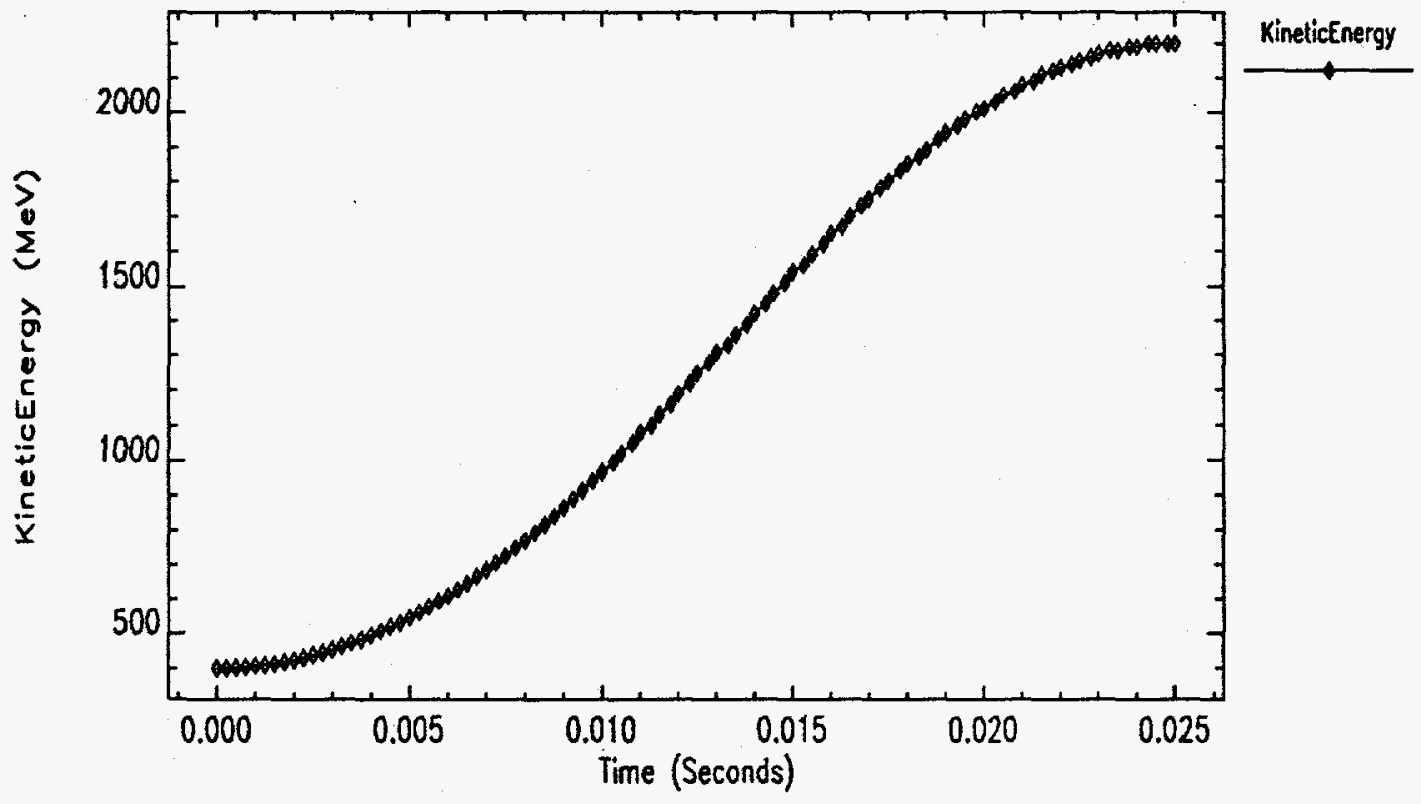

Figure 3: Neutralization during IPNS-II RCS acceleration cycle. 